\title{
Performance evaluation of IPTV traffic over pDSL
}

\author{
Diego L. Cardoso*, Marcelino S. da Silva, Marcos C. Seruffo, Lamartine V. de Souza, \\ Carlos R. L. Francês, Jorge A. M. de Souza, João C. W. A. Costa \\ Federal University of Pará, R. Augusto Côrrea, 01, 66075-110, Belém, PA, Brazil.
}

\begin{abstract}
The increasing trend of triple play traffic transmission (voice, video and data) over internet is a worldwide phenomenon. However, to get a satisfactory communication, we need study the process on end-the-end way. Into regions with little infrastructure of telecommunications, one of the concerns is about used technologies of access. This work presents an analysis based on an IPTV (Television Internet Protocol) transmission generated on two combined technologies of access, PLC (PowerLine Communications) and ADSL2+ (Asymmetric Digital Subscriber Line), using a combination of electric network and telephony infrastructure, called of pDSL. This technology is a viable combination for regions with poor infrastructure of telecommunications. The results are evaluated with intention to present the viability of this kind of the telecommunication environment and with a simulation for extrapolation study.
\end{abstract}

Keywords: Simulation, IPTV, pdsl, last mile.

\section{INTRODUCTION}

With the computer networks evolution, whose capacities of communication and transmissions guarantees are increasing substantially, new technological sources start to be established in the world-wide scene. The information technology evolution brings a trend of infra-structure barriers transposition so that can obtained positive results. With this, some technologies have being developed to follow the direction of this latent evolution, increasing the dissemination capacity of information.

In this direction, it is of basic importance that the used technologies have certain characteristics to provide real citizenship services and/or social digital inclusion. These characteristics, as including capillarity, low costs, implantation easiness and use, are important given that reaches regions with less of available infrastructure to the typical final user, which normally possess specific geographic characteristics, making difficult these solutions consolidation. Thus, it must always be pursued the possible technologies combinations that can enlarge the success possibilities of social behavior technologies. For that, it is essential to use "a priori" studies that present favorable relations between cost/benefit to determine telecommunications solutions. In this direction, technologies that inherited the available infrastructure from other technologies can diminish the implantation impact. Access technologies like ADSL2+ and PLC, for example, have its favor the fact of ADSL2+ be an access infrastructure already sufficiently consolidated in market, given that it uses the telephonic line for transmission of data; and PLC possess great capillarity, being considered as an ambiguous technology, since uses energy line for data transmission. Hence, a combining solution with PLC indoor and ADSL2+ is understood as the most viable network access to promote actions on digital inclusion in regions like Amazon.

This paper points a case study of IPTV traffic using pDSL combination, with intention to present measured results that prove the proposal solution viability. The choice for IPTV was to the fact of the strong social appeals and TV media strength possess in Brazil, reaching more than $90 \%$ of Brazilian homes, according to IBGE [1]. In [2] was carried through a study that describes the criteria for excellent performance of bandwidth allocation for links in pDSL networks.

The remain of this article is organized as fallow. In section II some concepts of IPTV are boarded. In section III the basic technological characteristics of ADLS2+, PLC and pDSL are presented. In section IV are argued about the implemented testbed. Section V presents the results obtained and the final section shows the conclusions.

*diego@ufpa.br; phone+55 91 3201-73021; lprad.ufpa.br

Broadband Access Communication Technologies II, edited by Raj Jain, Benjamin B. Dingel, Shozo Komaki, Shlomo Ovadia Proc. of SPIE Vol. 6776, 67760G, (2007) · 0277-786X/07/\$18 · doi: 10.1117/12.751924 


\section{IPTV}

IPTV service is the bilateral high speed internet service, is communication by IP packet, sends standard definition-level image, and provides triple play service including streaming image service. When IP TV sends image, voice, data contents, it uses MPEG. According to the high data speed, it uses BcN (Broadband convergence Network) when it delivers Contents [3], [4] and [5].

The IPTV service allows the use broadband access network to deliver TV content potentially increased of interactive services. Current IP networks offers a delivery packages service called "better effort", that does not offer performance guarantees for its users, in other terms, packages can be lost in its passage.

Delay, package loss and the bandwidth of networks are very important for the IPTV applications, at a time these networks can tolerate small packages losses, on the other hand impose severe temporization restrictions or minimum transmission capacity to guarantee a proper viability.

\section{ACCESS TECHNOLOGY}

For IPTV service supply, a technology that binds the final user to the final data-communication network (in this case, called last mile) becomes necessary.

The access to communication services normally is provided not by only one technology, but for a combination of them, what generates an infinity of problems, such as: different bandwidth, difficulties to guarantee Quality of Service (QoS), trustworthiness and scalability. Said that, it's important have a set of support decision tools to make the implantation planning of the existing technologies possible.

Currently, there are a lot of last mile technologies that can be used, and can be divided in two classes: (a) wired technologies: fiber optics (for last mile access and not to backbone); PLC; CATV (cable TV); xDSL and dialed line; (b) wireless technologies: cellular satellites, wireless local area networks (WLANs) .

\section{ADSL2+}

Digital Subscriber Line is a technology family that supplies digital data transmission, using the advantage of the telephony network that arrives in the majority of the residences. ADSL2+ it's a derivation from DSL, allowing data transmission faster than conventional ADSL.

DSL technologies possess a global abrangency, being considered as the dominant broad-band access technology not only in the Europe, as well as in Latin America and developing countries as India [6], [7] and [8]. In Latin America DSL technology is responsible for $77 \%$ of all broad-band access, in Brazil this value arrives $85 \%$. In quantitative terms, in the end of 2005, Latin America had almost 5.300.000 subscribers of ADSL technology [7].

Although such numbers, the broad-band services penetration in Latin America residences was about only $9 \%$ for the end of 2006, being projected about $17 \%$ for the and of 2015 [7]. In such a way, it can be understood that broad-band access services in Latin America have a long way to go.

\section{PLC}

The PLC technology uses the existing electric infrastructure to provide the data communication. This technology has shown itself sufficiently competitive in the broadband access market, disputing with other more traditional, like DSL. PLC counts with the advantage of having a sufficiently wide infrastructure and with relatively little cost associated [9].

PLC, however, as well as other data communication technologies, possesses some inconveniences. The physical environment is very hostile for the data, once that it was not conceived for this purpose, thus there are many properties of the power systems which influence negatively the high speed communications (losses in the cable, propagation in multiple paths and the noise, for example) [9] [10]. 
A way to reduce the impact of the transmission environment in the communication is the application of efficient modulation methods, such as OFDM (Ortogonal Frequency Division Multiplexing), besides mechanisms for error correction such as FEC (Forward Error Correction) and the ARQ (Automatic Repeat reQuest) [11].

However, independently of the adopted techniques, a factor to be observed and understood is the noise in the communication. The propagation of the signal through the power transmission line provokes an attenuation and a delay in the signal, which increases with the distance and the frequency. This attenuation depends on the impedance characteristic and on the propagation constant of the transmission lines. In accordance with [12] and [14], these two parameters are resistance functions, conductance, inductance, and capacitance, per unit of length, which depends on the frequency.

Beyond the environment inconveniences, other PLC barrier exists in what refers to the internet access. The service subscriber has an interface with the low tension network, it possess innumerable transformers throughout its extension. PLC signal must make a bypass on this equipment, aiming to keep the information integrity carried. Some PLC repeaters, for example, can be used to avoid the transformers. But depending on the network extension, the implantation investment of the service can increase substantially because of this inconvenience.

\section{PDSL}

Powerline Digital Subscriber Line is a high speed data communication solution that combines two technologies: ADSL and PLC. Its functioning is basically to supply multiple high speed broad-band links on force lines (power lines) in an indoor environment [2].

A pDSL network consists of a high speed links configuration between PLC Gateway and multiples pDSL devices. Such devices use channel training procedures to place bandwidth for each virtual link. In [15] was carried through a study that describes the criteria for excellent performance of bandwidth allocation for links in pDSL networks.

For interconnection between networks it was used pDSL gateways, that possess function of pDSL central communication and coordination unit, besides interface for external communications infrastructure (Figure 1).

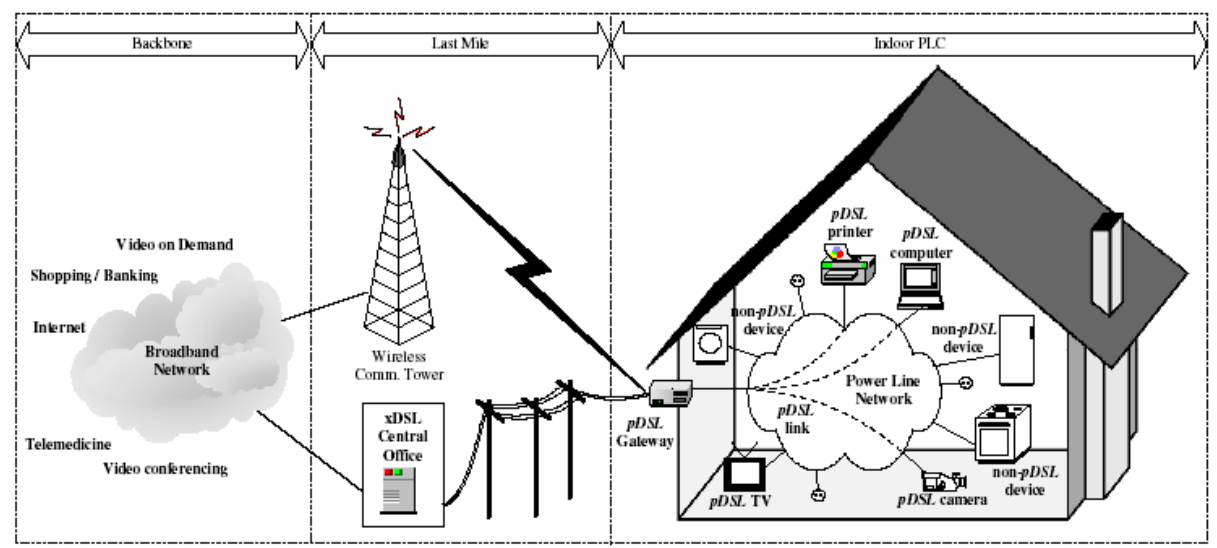

Fig. 1. pDSL Service Structure [11].

A indoor pDSL network has two device categories that are connected to its terminal point (electrical plug), they are: digital equipment that requires high speed transmission (known as pDSL devices), such as computers, printers and TVs; and devices that do not make use of such services, even using the same force line.

Figure 1 illustrates an example of a network using the indoor pDSL technologies, with pDSL and not pDSL devices connected to electric network. pDSL gateway establishes connection between the indoor network with another existing last mile technology (xDSL or wireless, for example). 


\section{FEASIBILITY STUDY BASED ON GAUGING OF THE SYSTEM}

\subsection{The scenes}

For experiment analysis, two testbeds were constructed in the LabIT (Laboratory of Technological Innovation in Telecommunications) of UFPA. The first one, showed in Figure 2, is composed by a DSLAM / EDA (xDSL network), PLC base station, PLC modems and a traffic generator (AX-4000), capable of generates diverse traffic types (UDP and TCP), making possible for a more deepened study of the pDSL technology behavior in high loaded situations.

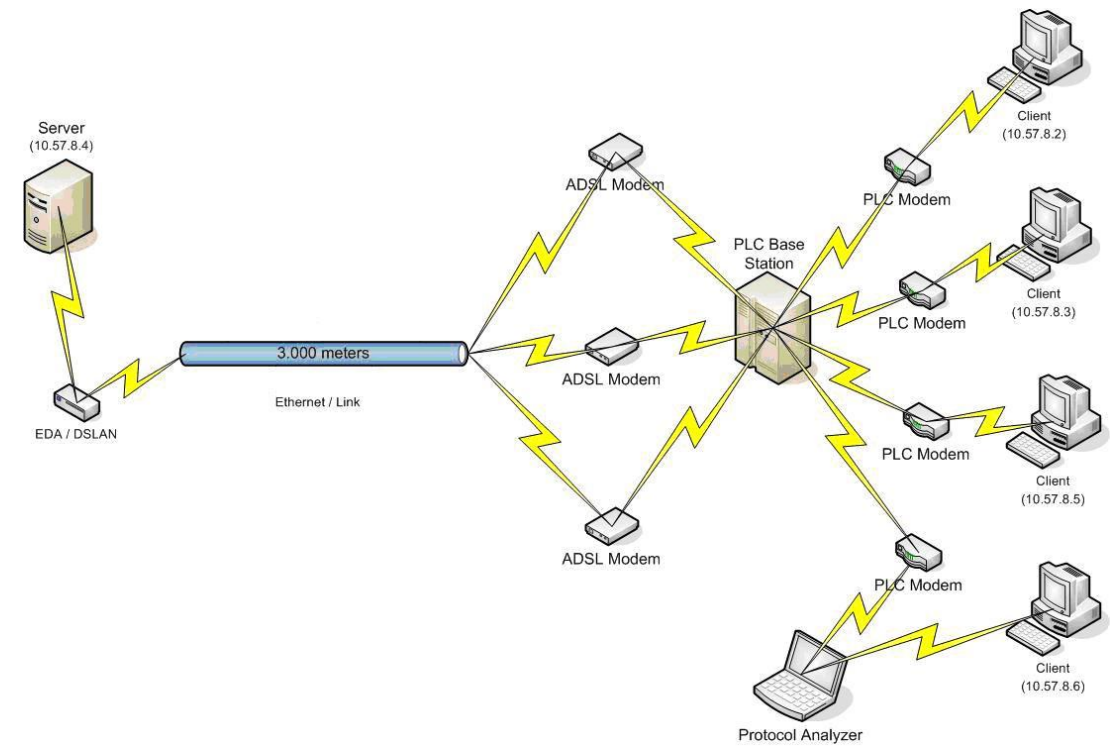

Fig. 2. Testbed of experiment I.

The second testbed showed in Figure 3, is a typical IPTV transmission. In this system, it have machines transmitting and receiving triple play streams. It uses ADSL modems, PLC modems, DSLAM, telecommunications cables, PLC base station, protocol analyzer, noise generator, client computers customer and one video server.

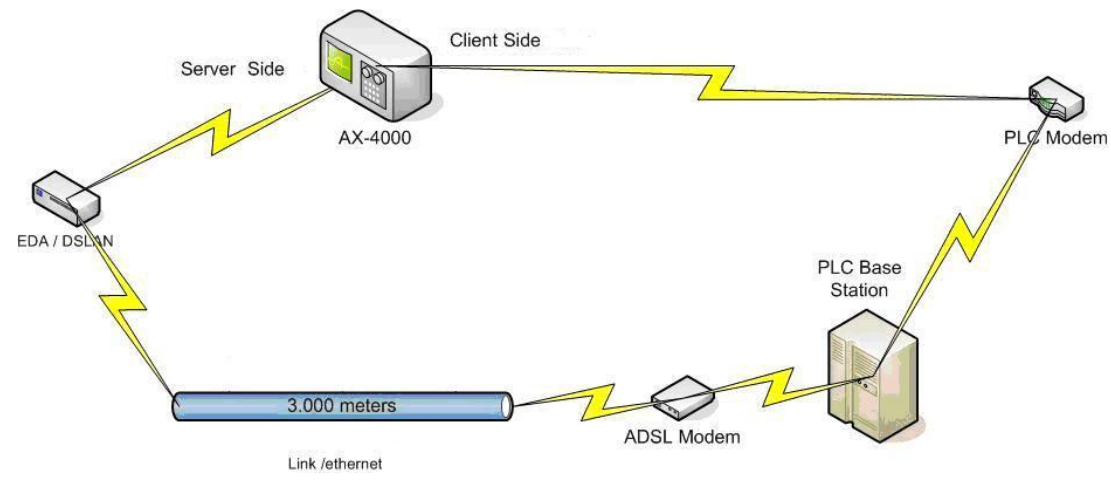

Fig. 3. Testbed of experiment II.

\subsection{Testbed Description}

DSLAM / EDA (Ethernet DSL Access) composes the existing equipment in the telephone central office side, allowing the communication using ADSL2+ links. The computer connected to DSLAM is responsible for generating video flows that will be distributed through multicast between the customers. As last mile technology, it was chosen to use real metallic cables, which were disposed around of the LEA/UFPA (Laboratory of Electric Engineering and Computation) 
building. For cables length determination, it was used Brazilian Standard Telebrás 225-540-788, 1997 April, which considers in the distance of 3.000 meters for one test link.

In the first testbed, for data traffic generation, it was used a benchmark called IPERF. A free software (Linux and Windows versions), used to simulate TCP connections (HTTP traffic), or UDP traffic - available in [16].

In video traffic generation, VLC was used (VideoLAN Client), which is a multimedia reproducer that supports big variety of video formats. For the video test, VLMS (VideoLAN MiniServer) was configured, a VLC streaming server, using multicast protocol, sending packages with a rate of $1 \mathrm{Mbps}$ for the three machines (video with MPEG2 codec) [17].

For the voice transport, Callgen was used, a VoIP tool developed by OpenH232 project and is available in [18], widely used for tests [19]. A RADCOM® protocol analyzer [20] was also used, filtering packages that will go pass through the network, isolating specific flows for performance measurement.

In the second testbed, $\mathrm{AX} / 4000{ }^{\circledR}$ was used, equipment developed by Spirent Communications possessing capacity of generate and analyze TCP/IP traffic. Besides traffic generation, AX/4000 can emulate ADSL2+ modems, allowing a detailed performance analysis of a DSL system.

\subsection{Measures for performance analysis}

Searching an appropriate study between the used technologies, were analyzed the measures: delay, jitter, MOS and packages loss. All metrics relative to voice transmission had been captured by the MediaPro, a module from RADCOM protocol analyzer, responsible for capturing and evaluating all VoIP calls made, informing MOS (Mean Opinion Score), jitter, package loss, amongst others metrics.

\section{RESULTS}

For each experiment, the results were studied and evaluated as exposed above. Both scenarios were analyzed in downstream and upstream data flow.

\subsection{Scenario I}

In this scenario, the AX-4000 equipment was used to generate the TCP data flow, with 1000 bytes long fixed packets. The data flow was used along the experiment with a maximum throughput (8Mbps for downstream ad 800kbps for upstream) to simulate a high load in the network in order to analyze these technologies in their limits.

To obtain a noise disturbance in PLC network two devices were added to the electrical network: an outlet surge protector and a battery charger [21] and [22]. Tables I and II shows the results obtained for downstream and upstream, respectively.

Table 1. Downstream performance evaluation (scenario I).

\begin{tabular}{c|c|c|c}
\hline & No disturbances & With surge protector & With battery charger \\
\hline Packets / s & 988 & 978 & 188 \\
\hline Average Delay (ms) & 16.055 & 16.754 & 16.058 \\
\hline Throughput (Mbps) & 7.907 & 7.823 & 1.507 \\
\hline Packet Loss Ratio & $8.18 \times 10^{-6}$ & $1.18 \times 10^{-4}$ & 0.355 \\
\hline
\end{tabular}

It is observed that after the addition of the outlet surge protector the influence of this device in the system is not so dangerous. However, when the battery charger is added to the system, the throughput of the network is decreased by $19 \%$ and the packet loss ratio increase, approximately, from 8 in one billion to 35 in one hundred. It occurs because the battery charger has more influence in the system than the outlet surge protector, as presented in [21]. 
Table 2. Upstream performance evaluation (scenario I).

\begin{tabular}{c|c|c|c}
\hline & No disturbances & With surge protector & With battery charger \\
\hline Packets / & 99 & 99 & 20 \\
\hline Average Delay (ms) & 43,083 & 43,344 & 53,543 \\
\hline Throughput (Mbps) & 0,7939 & 0,7937 & 0,16 \\
\hline Packet Loss Ratio & $7,17 \times 10^{-4}$ & $9,95 \times 10^{-4}$ & $4,33 \times 10^{-2}$ \\
\hline
\end{tabular}

Generally speaking, when the upstream data flow is observed, its behavior is similar to downstream. The outlet surge protector has no much impact in the metrics measured, but the battery charger results in a high loss of performance. The throughput decrease by $20 \%$ and the packet loss ratio increase from one in one thousand to 4 in one hundred approximately.

In both cases, upstream and downstream, the average delay is not so influenced by the disturbances.

Although this and the long distance between the DSLAN (in central office) and the ADSL2+ modem (3000 meters), this experiment shows that the communication can be viable. It depends on the devices connected in the system. It must be observed that the system is being stressed by the high traffic intensity of the IPTV application.

\subsection{Scenario II}

Table II shows a comparative of the results carried through the fifty VoIP calls. This VoIP calls were carried with and without background traffic (IPTV application). To analyze the influence of the other pDSL devices in the network an outlet surge protector was added to the system.

Table 3. Upstream performance evaluation (scenario II)

\begin{tabular}{c|c|c|c|c}
\hline & \multicolumn{2}{|c|}{ VoIP } & \multicolumn{2}{c}{ IPTV } \\
\hline Noise & Without & With & Without & With \\
\hline MOS & 1,194 & 1,145 & 1,196 & 1,165 \\
\hline Throughput (kbps) & 128,24 & 121,43 & 128,79 & 125,36 \\
\hline Delay (s) & 3,98 & 4,45 & 6,57 & 4,97 \\
\hline Jitter (ms) & 11,8 & 12,1 & 11,6 & 11,9 \\
\hline
\end{tabular}

Table 4. Downstream performance evaluation (scenario II)

\begin{tabular}{c|c|c|c|c}
\hline & \multicolumn{2}{|c|}{ VoIP } & \multicolumn{2}{c}{ IPTV } \\
\hline Noise & without & with & withou & With \\
\hline MOS & 4,2 & 4,2 & 4,2 & 4,2 \\
\hline Throughput (bps) & 81678,71 & 81662,27 & 81652,08 & 81684 \\
\hline Delay (s) & 130,7 & 138,8 & 1607,833 & 4094,182 \\
\hline Jitter (ms) & 0 & 0,6 & 4 & 1,4 \\
\hline
\end{tabular}

\section{SIMULATION STUDY}

In order to verify the feasibility of the pDSL technology for digital inclusion purposes, a fictitious scenario of a Regional Training Center (RTC) was used. These centers are used for the training of teachers, community people, and health 
agents of neighboring cities. These kinds of actions are common in Amazon due to its particular characteristics (cities distant from capital or many times with difficult access).

The RTC implantation is part of a set of governmental actions being made in the State of Pará, in partnership with the Power Suppliers of the North of Brazil S.A. (Eletronorte); which is making a high investment in network infrastructure, thus seeking ways to spread the education and the digital inclusion in currently unprovided areas. Using the Eletronorte fiber-optic backbone, an infrastructure could be created to provide high speed internet in distant cities (many times in the amidst forests), which would need a last mile technology to receive these information; fact that motivated this paper. This way, government agencies, schools, RTC and users in general could be benefited.

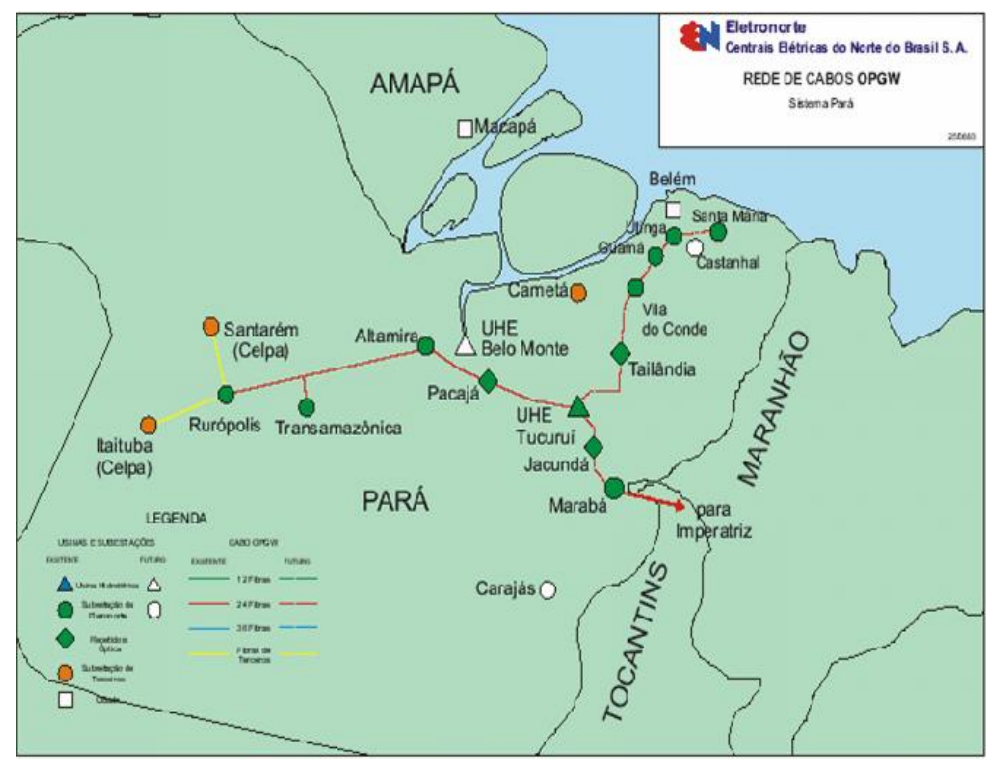

Fig. 4. Eletronorte backbone considered.

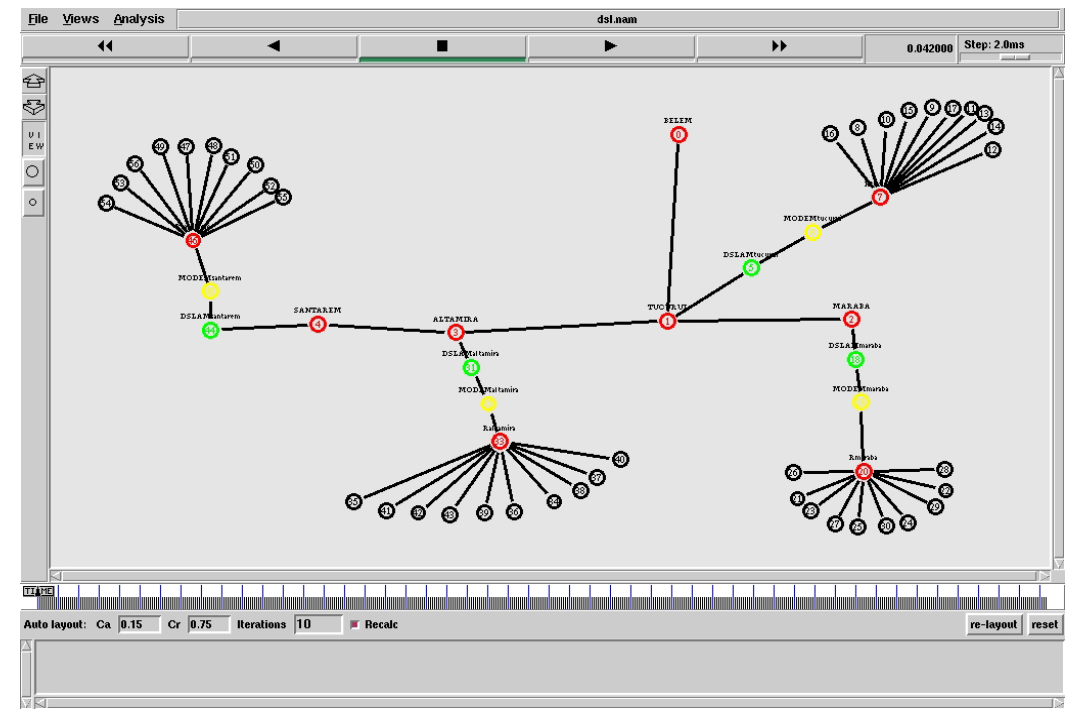

Fig. 5. NS-2 Simulation.

A RTC is composed by one computer laboratory, with ten (10) computers, being one of them connected to the Internet using indoor PLC and ADSL2+ last mile technology (pDSL). Similar RTCs laboratories would be distributed around the State of Pará. 
The laboratories are destined for video conferencing lectures, internet access and VoIP applications; Figure 4 shows a pDSL-based infrastructure of a RTC.

Four scenarios were proposed for scalability system analysis, each scenario has a video being multicasted for all RTCs. One client per city is making a VoIP call to another city and all computers are accessing internet. The difference between the scenarios is the available bandwidth: (a) 300Kbps of downstream and $150 \mathrm{Kbps}$ of upstream; (b) $600 \mathrm{Kbps}$ of downstream and $300 \mathrm{Kbps}$ of upstream; (c) $1 \mathrm{Mbps}$ of downstream and $600 \mathrm{Kbps}$ of upstream; (d) $8 \mathrm{Mbps}$ of downstream and $1 \mathrm{Mbps}$ of upstream.

The input data set was obtained from a trace file captured by a RADCOM( $\odot$ Performer Analyzer used during the experiments (scenario I and scenario II).

Table 5. Data of Video (Values gotten in average)

\begin{tabular}{c|c|c|c|c}
\hline & \multicolumn{4}{|c}{ Bandwidth } \\
\hline Video & $300 \mathrm{Kbps}$ & $600 \mathrm{Kbps}$ & $1 \mathrm{Mbps}$ & $8 \mathrm{Mbps}$ \\
\hline Delay (s) & 0,677 & 0,084 & 0,065 & 0,046 \\
\hline Throughput (Kbps) & 246,533 & 255,653 & 255,653 & 255,789 \\
\hline Jitter (s) & 0,044 & 0,021 & 0,012 & 0,005 \\
\hline Lost Package (\%) & 0,215 & 0,187 & 0,189 & 0,187 \\
\hline
\end{tabular}

Table 6. Data of VoIP (Values gotten in average)

\begin{tabular}{c|c|c|c|c}
\hline & \multicolumn{4}{|c}{ Bandwidth } \\
\hline VoIP & $300 \mathrm{Kbps}$ & $600 \mathrm{Kbps}$ & $1 \mathrm{Mbps}$ & $8 \mathrm{Mbps}$ \\
\hline Delay (s) & 1,404 & 0,253 & 0,084 & 0,071 \\
\hline Throughput (Kbps) & 48,826 & 51,935 & 51,834 & 50,811 \\
\hline Jitter (s) & 0,050 & 0,033 & 0,015 & 0,005 \\
\hline
\end{tabular}

Objecting evaluate the results obtained, a table of bordering values obtained in literature was used as comparative parameter, which presents a series of values for data applications, voice and video. Such values are demonstrated in Table 7

Table 7. QoS values of applications

\begin{tabular}{|c|c|c|c|}
\hline Parameter & Voice Service & Data Service & Áudio/Vídeo Service \\
\hline PDT_V1 & $100 \mathrm{~ms}$ & $1000 \mathrm{~ms}$ & $100 \mathrm{~ms}$ \\
\hline PDT_V2 & $400 \mathrm{~ms}$ & $4000 \mathrm{~ms}$ & $400 \mathrm{~ms}$ \\
\hline PDV_V1 & $1 \mathrm{~ms}$ & $10 \mathrm{~ms}$ & $1 \mathrm{~ms}$ \\
\hline PDV_V2 & $100 \mathrm{~ms}$ & $100 \mathrm{~ms}$ & $100 \mathrm{~ms}$ \\
\hline THRU_V1 & $64 \mathrm{kbps}$ & $10 \mathrm{kbps}$ & $128 \mathrm{kbps}$ \\
\hline THRU_V2 & $10 \mathrm{kbps}$ & $1 \mathrm{kbps}$ & $10 \mathrm{kbps}$ \\
\hline PLR_V1 & $10^{-4}$ & $10^{-6}$ & $10^{-4}$ \\
\hline PLR_V2 & $10^{-3}$ & $10^{-4}$ & $10^{-3}$ \\
\hline
\end{tabular}


where:

\author{
PDT: Packet Delay Transfer; \\ PDV: Packet Delay Variation, known as Jitter; \\ THRU: Throughput; \\ PLR: Packet Loss Rate; \\ V1: Inferior limit; \\ V2: Superior limit.
}

All results obtained indicate pDSL as a viable last mile technology for IPTV applications

\title{
10. CONCLUSION
}

The utilization of the infrastructure of the user residence decreases the costs of the service, and can reach significantly quantity of potentially end users of the services. It can be a differential between to prove or not a service in regions with few infra-structure of communications (like in Amazon region). This work presents results of a experimental analysis for the transmission of high traffic intensity of IPTV in presence and absence of noise, with usage of indoor PLC net and for the solution of last mile the usage of ADSL2+ (of the xDSL family).

The unsatisfactory results of upstream scenario II can be explained with the length of the communication link ADSL2+ of test. The distance where based in Telebrás norm 225-540-788 of april 1997, which is inadequate as reference for these tests, in the sake that newer norms [23] indicate that a maximal distance for test communication links have to be in order of $2500 \mathrm{~m}$. This way, the values of reference taken from the norms for this experiment, when submitted for extern situations, as of Amazônia land, presents some difficulties that can not be rejected in the access nets project on penalty of make the traffic of some applications with QoS requirements in determined areas impracticable.

The presented results of experiments and simulation lead a conclusion that the use of a pDSL solution of last mile (using ADSL2+ and indoor PLC) presents satisfactory results.

\section{ACKNOWLEDGMENT}

Authors would like to thank the PROCAD (Program of Academic Cooperation between Universities) and CNPq (National Conceal of Scientific and Technological Development). Authors would like to thank the Research and Development Centre, Ericsson Telecomunicações S.A., Brazil, Federal University of Pará, Department of Electrical and Computing Engineering, Belém, Pará, Brazil. And Ericsson AB, Access Signal Processing Laboratory, Älvsjö, Sweden. Some of the authors want to acknowledge the financial support received from the European Commission IST 6th Framework and from the Swedish Agency for Innovation Systems, VINNOVA, through the IST - MUSE and the Eureka - Celtic BANITS projects respectively, which partially enabled this work.

\section{REFERENCES}

1. Available at http://www.ibege.gov.br, accessed 05/05/2007.

2. Antonakopoulos, T.; Papandreou, N. "Subchannels Allocation on Multiple pDSL Lines". Power Line Communications and Its Applications, International Symposium on.2005.

3. Seong, H. K. and Gil, H. L. (2006). "The Study on SLA Metrics and System Architecture for IP TV". In: The 8th International Conference on Advanced Communication Technology (ICACT2006), Gangwon-Do, Korea.

4. Al-Hezmi, A.; Rebahi Y.; Magedanz T.; Arbanowski S. "Towards an Interactive IPTV for Mobile Subscribers". In: ICDT/IEEE International Conference on Digital Telecommunications.2006.

5. Wu, J., Peng, K., Lu, M. Lin, C., Cheng, Y., Huang, P., Yao, J., Cheng, H. H. (2006) "HotStreaming: Enabling Scalable and Quality IPTV Services", In: IPTV Workshop, International World Wide Web Conference. May 23, Edinburgh, Scotland, United Kingdom. 
6. Olsen, B.; Katsianis, D.; Varoutas, D.; Stordahl, K.; Harno, J.; Elnegaard, N.; Welling I.; Loizillon, F.; Monath, T.; Cadro, P. "Technoeconomic Evaluation of the Major Telecommunication Investment Options for European Players", IEEE Network, vol. 20, issue 4, pp.6-15, July/August 2006.

7. Arenas, D.; Caldas, C.; Ramundo, C.; Vargas, S.; Hostos, L. "Challenges to expanding Fixed Broadband Services in Latin America", White Paper, Alcatel Telecommunications. September 2006.

8. Faudon, V.; Vleeschauwer, D.; Festraets, E.; Ross, P. . "End-User Services for Broadband uptake in High-Growth Economies", White Paper, Alcatel Telecommunications. September 2006.

9. Meng, H. e Guan, Y. L. "Modeling and Analysis of Noise Effects on Broadband Power-Line Communications", In: IEEE Transactions on Power Delivery, Vol. 20, No. 2, Abril 2005.

10. Zimmermann, M., Dostert, K. "Analysis and Modeling of Impulsive Noise in Broad-Band Powerline Communications", In: IEEE Transactions on Electromagnetic Compatibility, Vol. 44, No. 1, Fev. 2002.

11. Hrasnica, H., Haidine, A., Lehnert, R. "Broadband Powerline Communications Networks - Network Design", John Wiley \& Sons, Inc, 2004.

12. Zimmermann, M., Dostert, K. The low voltage power distribution network as last mile access network-signal propagation and noise scenario in the HF-range" In: AEÜ Int. J. Electron. Commun., Vol. 54, No. 1, 2000.

13. Andreou, G. T., Manitsas, E. K., Labridis D. P., et al "Finite element characterisation of LV power distribution lines for high frequency communications signals", In: $7^{\circ}$ International Symposium on Power-Line Communication and its Applications (ISPLC), Kyoto, Japão, 2003.

14. Hooijen, O. "A channel model for the residential power circuit used as a digital communications medium" In: IEEE Transactions on Electromagnetic Compatibility, Vol. 40, No. 4, November 1998.

15. Anastasiadou, D.; Antonakopoulos, T. "Broadband Communications in the Indoor Power Line Environment: The pDSL Concept". n Proc. ISPLC'04, Zaragoza, Spain, March 2004, pp. 334--339. (2004). Available at http://citeseer.ist.psu.edu/anastasiadou04broadband.html. Accessed 28/04/2007.

16. Iperf - The TCP/UDP Bandwidth Measurement Tool. Available at http://dast.nlanr.net/Projects/Iperf/, accessed $02 / 05 / 2007$.

17. VLC (2007). "VLC Media PLayer". Available at www.videolan.org/vlc, accessed 10/01/2007.

18. OpenH323. (2007) Available at http://www.openh323.org/, accessed 10/01/2007.

19. Mann, I.; McLaughlin, S.; Henkel, W.; Kirkby, R.; Kessler, T. "Impulse Generation with Appropriate Amplitude, Length, Inter-Arrival, and Spectral Characteristics", IEEE Journal on Selected Areas in Communications. Vol. 20, n. 5, pp. 901-912, June 2002.

20. Radcom. (2007) "The State of Art". Available at http://www.radcom.com/, accessed 01/02/2007.

21. Souza, Jorge Antônio Moraes de; SILVA, Marcelino Silva da; FRANCES, Carlos Renato Lisboa; COSTA, João Crisóstomo Weyl Albuquerque da; SEGATTO, Marcelo E V; ANTÔNIO, Flávio R; RODRIGUES, Gabryella, "A feasibility study of powerline communication technology for digital inclusion in Brazilian Amazon". In: SPIE OPTICS EAST. 2006, Boston-USA.

22. Souza, J. A. M., Silva, M. S., Francês, C. R. L., Costa, J. W., Segatto, M. E. V., Antônio, F. R., Vijaykumar, N. L. "A Feasibility Study of PLC Technology for Digital Inclusion". VI International Telecommunications Symposium (ITS2006), Fortaleza, Brazil, 2006.

23. TR-100, ADSL2/ADSL2plus Performance Test Plan, DSL Forum, March 2007. Rivers, http://awebsiteref.com 